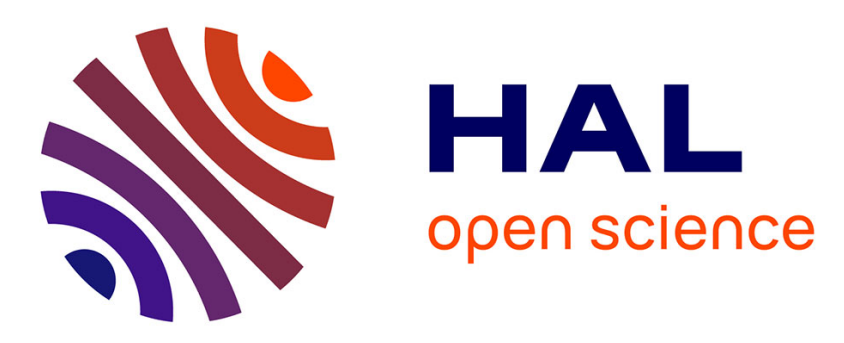

\title{
Architecture de systèmes contrôlés par la produit pour un environnement de juste à temps
}

André Thomas, Hind El Haouzi, Thomas Klein, Sana Belmokhtar, Carlos

Herrera

\section{- To cite this version:}

André Thomas, Hind El Haouzi, Thomas Klein, Sana Belmokhtar, Carlos Herrera. Architecture de systèmes contrôlés par la produit pour un environnement de juste à temps. Journal Européen des Systèmes Automatisés (JESA), 2009, 43 (4-5), pp.513-535. 10.3166/jesa.43.513-535 . hal-00542384

\section{HAL Id: hal-00542384 \\ https://hal.science/hal-00542384}

Submitted on 2 Dec 2010

HAL is a multi-disciplinary open access archive for the deposit and dissemination of scientific research documents, whether they are published or not. The documents may come from teaching and research institutions in France or abroad, or from public or private research centers.
L'archive ouverte pluridisciplinaire $\mathbf{H A L}$, est destinée au dépôt et à la diffusion de documents scientifiques de niveau recherche, publiés ou non, émanant des établissements d'enseignement et de recherche français ou étrangers, des laboratoires publics ou privés. 


\title{
Architecture de systèmes contrôlés par la produit pour un environnement de juste à temps
}

\author{
André Thomas - Hind El Haouzi - Thomas Klein - Sana Belmo- \\ khtar - Carlos Herrera
}

Centre de Recherche en Automatique de Nancy,

Nancy Université - CNRS (UMR 7039)

Andre.Thomas@cran.uhp-nancy.fr

\begin{abstract}
RÉSUMÉ. Apparu dans les années 80, le juste à temps (JiT) a permis plus de flexibilité et de réactivité, tout en conduisant à une meilleure maîtrise des stocks d'en-cours. L'application de cette philosophie donne des possibilités de prise de décision autonomes à l'atelier, donnée en général aux opérateurs. Le principe de Système Contrôlé par le Produit, issu du paradigme holonique, est encore émergeant et vise à donner aux produits des capacités d'information, voire de prise de décision. Nous montrons dans cet article que son application dans le contexte particulier d'entreprises gérées en JiT est pertinente à des fins d'aide à la décision ou d'automatisation des actions des opérateurs. Une architecture de contrôle est proposée comprenant un système de contrôle, un émulateur, une base de données et des interfaces adaptées. Deux applications industrielles sont aussi présentées. En terme de perspectives nous initions une problématique d'intégration d'un module d'optimisation en ligne dans cette architecture. ABSTRACT. Appeared in 80's, Just in Time (JiT) leads to more flexibility, reactivity and more Work in Process control. This philosophy gives autonomous decision making possibilities to shop floor operators. Coming from holonic manufacturing paradigm, Product Driven System (PDS) Concept is still emergent and leads to give information and decision capacities to products. In this paper, we show that in the specific JiT context, PDS are pertinent for decision making process and operational tasks automation. A control architecture is also proposed. This architecture is composed by a control system, an emulator, a data base and interfaces. Two industrial applications are presented. In perspectives, we initiate the optimisation module integration problem.

MOTS-CLÉS : Juste à temps, Systèmes Contrôlés par le Produit, simulation, émulation

KEYWORDS: Just In Time, Product Driven Systems, Simulation, Emulation
\end{abstract}

$1^{\text {re }}$ soumission à JESA, le 26 janvier 2009 


\section{Introduction}

Depuis les années 80 nous avons vu se développer dans les industries diverses applications des concepts du juste à temps (JiT). Les systèmes de production et de logistique ont considérablement évolués pour assurer plus de réactivité, plus de flexibilité. De systèmes centralisés et hiérarchisés pilotant entièrement les flux physiques nous sommes passés à des modes de contrôle « hybrides » donnant aux opérateurs de terrain plus d'autonomie et plus de responsabilités (les systèmes kanban en sont un exemple bien connu). Nous avons donc migré vers des systèmes où la décision autonome et locale, prise par les opérateurs essentiellement, est devenue possible. Ceci a permis, entre autre, de supprimer des niveaux de prise de décision dans le système centralisé, de simplifier les processus de contrôle en évitant de nombreuses boucles de retour d'information (et donc d'améliorer la réactivité ou la vitesse de prise de décision face à des perturbations), de simplifier aussi les nomenclatures (car de nombreuses étapes des processus de transformation n'étaient plus gérées par le MRP et donc disparaissaient de celles-ci) et d'alléger par conséquent les bases de données. Par la suite, on a constaté un manque de visibilité : les décisionnaires de production et de logistique pilotaient alors des «boîtes noires » n'en contrôlant que les entrées et sorties, car les opérateurs de manière autonomes modifiaient les plans prévus sans leur demander leur avis. Nous avons observé dans les entreprises aussi un manque d'optimalité : les opérateurs ne prenant des décisions qu'à partir d'une vision partielle du système, il est donc impossible d'assurer un taux de service client ou un taux de charge d'un goulot, par exemple, (Scholz-Reiter et al., 2008). Dans la mouvance des initiatives liées au paradigme Integration in Manufacturing (IiM) (Burbidge et al., 1987), une forme d'automatisation de ces processus de terrain est alors apparue depuis quelques années afin d'assurer de la visibilité, de la tracabilité et de fournir une certaine aide à la décision locale, voire des propositions d'optima. L'apparition des nouvelles technologies d'information et de communication, telles les RFID, devrait permettre à terme de changer le processus classique de gestion de la production.

Plus dernièrement, la communauté de l'Intelligent Manufacturing Systems (IMS) (Babiceanu et al., 2006) a beaucoup soutenu les initiatives conduisant à donner au produit ou à des éléments du système physique des capacités de portage d'information, voir de traitement de celles-ci. Parmi ces principes d'automatisation, le concept de « système contrôlé par le produit » $(\mathrm{SCP})$ semble novateur dans la mesure où il confère directement au produit lui-même des capacités lui permettant d'assurer les fonctionnalités palliant aux problèmes précédemment cités. Par opposition au classique MRP2, système centralisé et hiérarchisé, le concept de SCP est une manière différente de voir le contrôle d'un atelier ou, d'une manière générale, des flux physiques de produits et de matières. Selon ce concept, le produit peut porter, de manière permanente et continue (ou de manière discontinue), des informations. Il peut aussi en donner à et/ou en capter de son environnement immédiat. Il peut enfin sur la base de ces informations, et faisant suite à un traitement qu'il opérerait lui-même, prendre des décisions relatives à son propre devenir et les faire appliquer par les éléments constitutifs de son environnement. Ce concept a transformé la boucle de contrôle cybernétique (Figure 
1) et a été largement présenté dans le passé (Valckenaers et al., 1997), (Bongaerts et al., 2000),(Brennan, 2000) (Morel et al., 2007) et (Cavalieri et al., 2003).

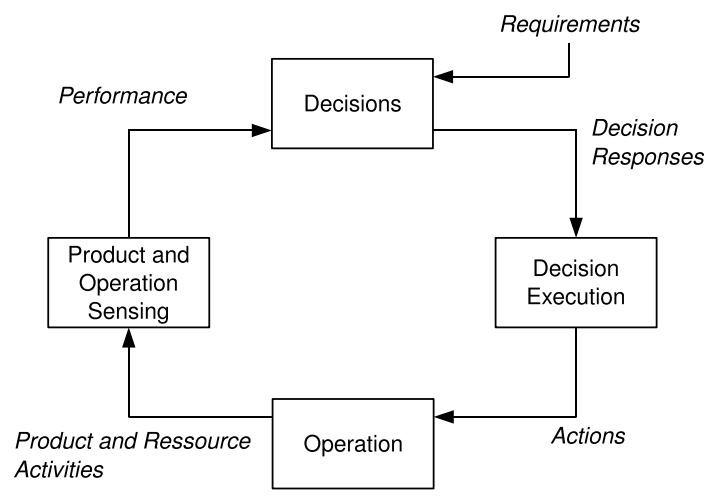

Figure 1. Boucle de contrôle incluant le produit informant

L'objectif de cet article est de montrer en quoi ce concept peut être particulièrement pertinent dans un contexte de JiT et quelles architectures sont alors nécessaires pour permettre aux divers acteurs du processus de décision de piloter dynamiquement les flux physiques. En s'appuyant sur une description de deux cas industriels, nous introduirons le concept de «Systèmes Contrôlés par le Produit » (SCP). Nous spécifierons l'architecture de contrôle proposée. Puis la dernière partie décrira l'application envisagée dans les deux entreprises. Enfin nous conclurons et proposerons des perspectives qui s'appuient sur les limites de cette proposition.

\section{Illustrations par deux cas industriels}

\subsection{Une entreprise de meubles en kanban}

Le cas d'étude considéré est un atelier de fabrication de meubles en kits d'une usine appartenant à un groupe industriel français. Cet atelier est composé de onze centres de charge, regroupés en quatre îlots (Figure 2). Le premier îlot, de débit, représente un processus disjonctif de découpe de grands panneaux en pièces. Chacun des centres de charge est doté de fonctionnalités différentes et peut réaliser plusieurs opérations. Par conséquent, de nombreux chemins sont possibles, et la complexité des flux est accrue par la diversité et le volume de pièces : environ 70 lots de 400 pièces sont lancés quotidiennement. L'opération terminale est l'emballage (Ilot 4) des différentes colis, où les pièces sont regroupées par référence de colis. Cette opération représente une convergence pour laquelle les flux de composants doivent être re-synchronisés, et de ce fait, est le goulot du système. La grande quantité de références (1800 références meubles, soit plus de 10000 références pièces) et la diversité de celles-ci induisent 
également une grande complexité des flux. Le système est, au moment de l'écriture de cet article, géré à l'aide d'un logiciel d'ordonnancement prédictif (Ortems), dont le but est de respecter un programme directeur de production établi sur prévisions. A ce moment du début de l'étude, l'entreprise analyse l'opportunité de basculer vers un mode de production juste-à-temps, avec une gestion des flux de production à l'aide d'un système inspiré du Kanban (nommé « kanban1 » dans la partie 5) dans lequel les opérateurs devraient gérer les priorités entre les lots.

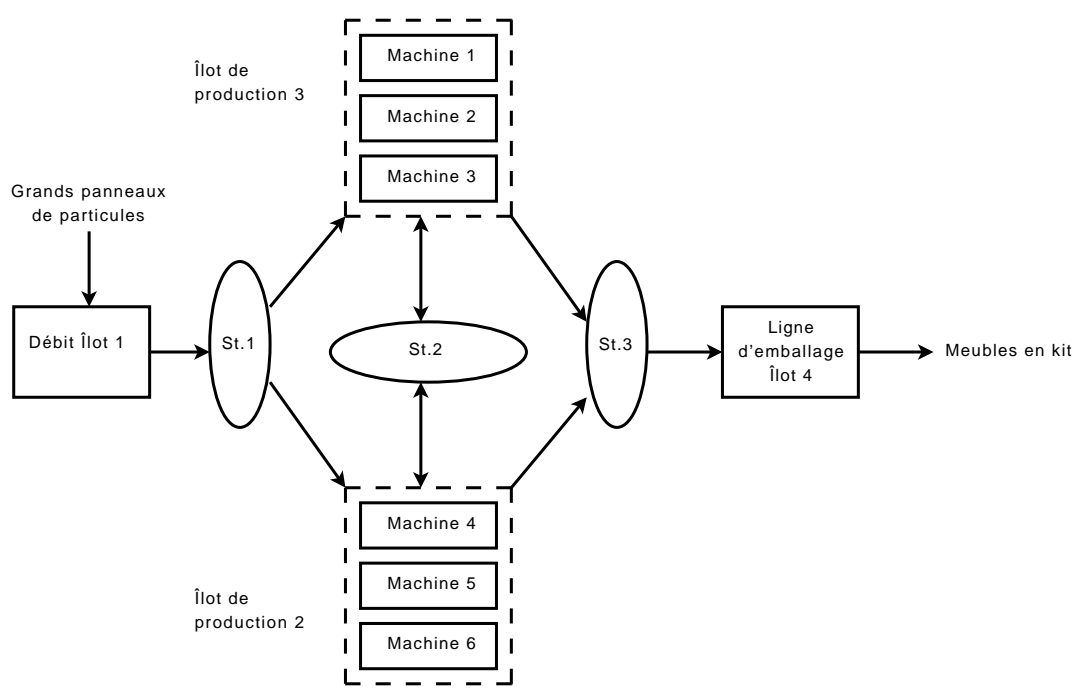

Figure 2. Vue synthétique du système de production considéré

\subsection{Un fabricant de systèmes de climatisation en DFT}

La deuxième entreprise appartient à un groupe américain. Elle propose une large gamme de produits de l'industrie du froid allant du simple climatiseur au système réfrigérant pour usine agro-alimentaire. Cette entreprise possède 27 sites de production dans le monde, toutes ses usines sont organisées de la même manière, à savoir la DFT (Demand Flow Technology) (Costanza, 1996). Cette méthode est une mise en œuvre du juste à temps et permet de standardiser les différentes processus physiques et décisionnels dans une entreprise. Elle s'appuie sur la demande du client et par une détermination formelle permet de concevoir des lignes d'assemblage répondant à cette demande. Cependant, cette méthode ne permet pas de prendre en compte le caractère dynamique de la ligne; par exemple, des files d'attente qui peuvent se créer à cause du déséquilibre de charge entre les différentes postes d'une ligne et à cause des problèmes liés à la synchronisation des flux matières entre une ligne d'assemblage et ses lignes d'approvisionnement. Il devient donc nécessaire de prendre localement diverses dé- 

postes de la ligne principale.

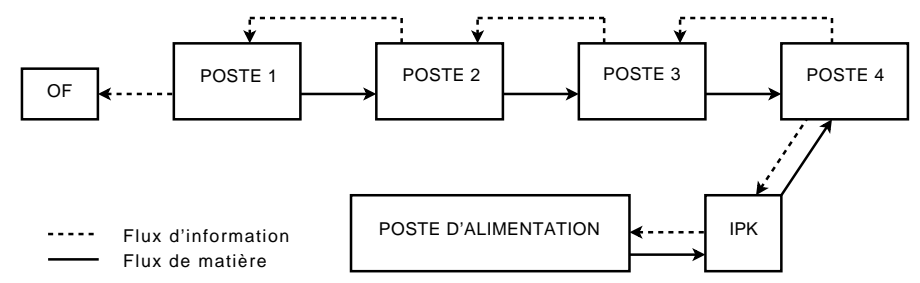

Figure 3. Le mécanisme de synchronisation

La synchronisation entre une ligne d'assemblage et ses lignes d'alimentation se fait, pour éviter toute rupture, grâce à des signaux visuels, concretisés par un emplacement de stock dit «IPK » (In Process Kanban) qui est prévu entre la ligne d'assemblage et le poste d'alimentation (Figure 3), la capacité de cet emplacement est variable (de 1 à n produits). Cet IPK fonctionne, en fait, comme un kanban (l'appellation « kanban physique » est quelquefois utilisée dans la littérature) : une consommation dans celui-ci entraîne un appel de recomplètement pour la ligne d'alimentation. Dans ce cas d'étude, l'entreprise a donc installé un système JiT (DFT), mais est consciente d'un certain nombre de difficultés résiduelles de contrôle, entre autres, la minimisation des volumes de produits (qui peuvent être très volumineux) aux IPK.

\section{Points communs et singularités}

\subsection{Points communs}

Dans ces deux entreprises des formes de JiT sont (cas 2) ou vont (cas 1) être mises en place. Des décisions locales (à même le flux) et indépendantes du système de planification centralisé, sont prises dans un cadre d'autonomie défini cependant par ce dernier. La performance du système de production se mesure à l'issue de celui-ci par des indicateurs tels que la productivité globale de la ligne, le taux de service client ou le taux de charge des goulets d'étranglement.

\subsection{Singularités}

Il s'agit de décisions locales autonomes sur lesquelles nous avons choisi de travailler pour montrer l'intérêt d'un SCP. Dans le premier cas les opérateurs font «avancer » les produits grâce aux étiquettes kanban en respectant un ordonnancement à capacité finie défini pour la journée par le système centralisé. A l'intérieur de cette journée ils pourront gérer diverses priorités de passage des lots sur les machines en 
fonction des avances/retards que ces lots pourraient avoir pris ou des indisponibilités ressources qui pourraient survenir. Dans le deuxième cas, les opérateurs ont à gérer le respect strict du temps de cycle par leur mobilité d'un poste à l'autre, d'une part, et à gérer la synchronisation des lignes d'alimentation avec la ligne principale en cas d'aléas sur l'une de ces lignes, d'autre part. C'est sur ce deuxième aspect que nous avons choisi de focaliser notre application.

\section{Les systèmes contrôlés par le produit}

\subsection{Concept}

Ce concept s'appuie sur une interprétation du paradigme Holonic Manufacturing Systems (Koestler, 1976) pour expérimenter l'intérêt de rendre le produit, et au-delà l'ensemble du procédé, interactifs afin d'organiser de façon plus collaborative l'interopérationnalité des différentes systèmes hétérogènes de contrôle et de gestion (APS, ERP, MES) composant la chaîne de production et de logistique des entreprises. Cette interprétation tire parti des progrès et de la miniaturisation croissante des technologies infotroniques (RFID, communications sans-fils, etc.) et mécatroniques (composants logiciels embarqués) pour faire de ce produit actif un objet composite assurant une relation récursive logiciel-matériel entre les services et les biens associés aux produits en reliant tout objet logique de contrôle ou de gestion à au moins un objet physique du procédé. Ce paradigme SCP, qui a conceptuellement guidé l'ensemble des travaux du laboratoire (Morel et al., 2003), (Gouyon, 2004), (Pannequin et al., 2004), (Morel et al., 2007) (Figure 4) est une alternative pragmatique pour permettre aux entreprises de migrer d'une organisation intégrée et centralisée vers une organisation adaptable, puisque l'originalité de cette approche est de combiner des décisions centralisées prises a priori sur des horizons à moyen, voire à long termes, avec des décisions décentralisées prises « en exécution » sur des horizons à court terme, voire sur événements.

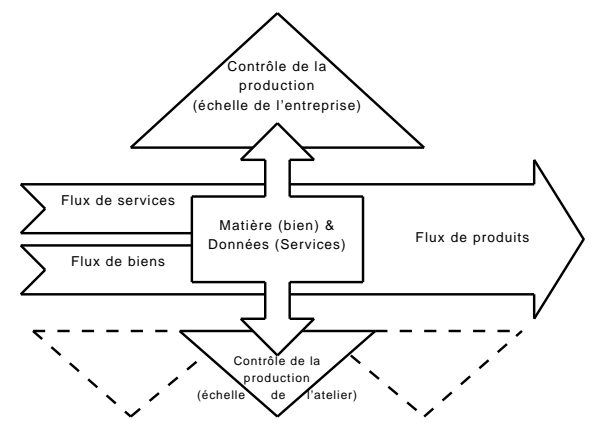

Figure 4. B2M interaction centered on holonic products (Pannequin et al., 2004 adapté de Morel et al., 2003). 
Nos travaux visent donc à mettre, au moins partiellement, en application ce concept. Ceci peut-être effectivement réalisé dans les cas industriels présentés précédemment dans la mesure où des décisions humaines sont transférées, grâce aux technologies de communication sans fil, sur le produit ou sur un élément du système physique (kanban) appartenant aux flux de produits. Se pose alors le problème de l'architecture du système informatique support.

\subsection{Architecture proposée}

Dans cette partie, nous allons décrire l'architecture proposée. Elle se veut adaptable à divers cas d'application relevant du domaine précédemment décrit. Elle doit à la fois permettre d'accompagner un projet de conception-intégration d'un SCP et permettre de piloter en phase d'exploitation un tel système conçu. Pour ce faire cette architecture devra aider à faire des choix technologiques en prenant en compte la dynamique du système réel (les files d'attente, la mobilité des hommes dans le système, les temps de réaction, etc.). Elle devra encore être mise en place, pour un cas industriel donné, dans un minimum de temps. Ainsi et compte tenu de ces objectifs évidents de réutilisabilité et de modularité (El Haouzi et al., 2005), (Pannequin et al., 2004), elle s'appuie donc sur un modèle d'émulation représentatif de l'atelier de production considéré et un système de contrôle de l'exécution qui intègre l'ensemble des règles de prise de décision. L'interface, basée sur un système d'information et une standardisation des interactions, permet l'interopérabilité et la synchronisation du modèle d'émulation et du système de contrôle. Cette architecture est également composée d'une bibliothèque de scénarii de test, et d'un module d'analyse a posteriori des résultats de simulation (Figure 5).

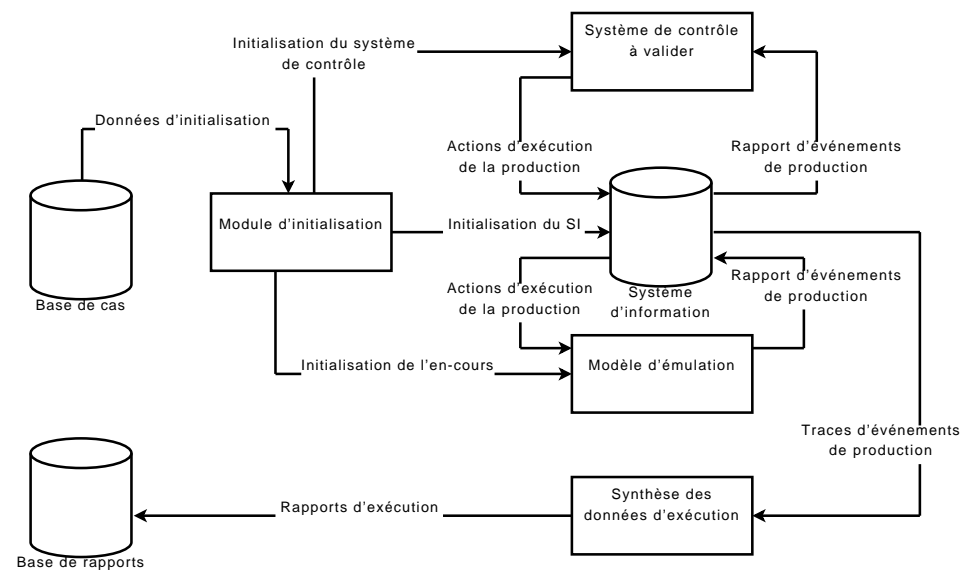

Figure 5. Modèle général d'architecture de contrôle centré sur le produit, Klein et al., 2006. 


\subsection{Modèle d'émulation}

L'objectif du modèle d'émulation est de représenter le comportement des éléments physiques du système opérant, tant les infrastructures comme les machines ou les engins de manutention que les flux de produits. Il n'intègre aucune règle de décision : chaque point où une décision est requise est transformé en un point de synchronisation entre modèle d'émulation et système de contrôle. Le modèle d'émulation est une représentation descriptive du procédé basé sur les principes de la systémique (Le Moigne, 1977). Compte tenu de notre point de vue (nous cherchons à modéliser un système de production), trois transformations élémentaires nous permettent de modéliser l'ensemble des comportements :

- Transformation forme/temps : représente une transformation physique du produit à travers le temps. Utilisé pour représenter une opération comme un usinage, un perçage, etc., dans un système de production.

- Transformation espace/temps : représente une transformation d'espace à travers le temps, c'est à dire le déplacement d'une entité. Typiquement utilisé pour représenter une opération de transport.

- Transformation de temps : représente tout stockage. L'entité n'est pas sujette à transformation ou transport durant une période de temps.

Par ailleurs, deux relations de composition permettent de représenter deux transformations complémentaires :

- Assemblage : représente l'assemblage de plusieurs entités en une seule.

- Désassemblage : représente la division d'une entité en plusieurs, qui peuvent être identiques ou différentes. Utilisé pour modéliser des opérations comme la découpe.

Des primitives génériques de modélisation ont ainsi été développées. Une fois le process modélisé à l'aide de ce formalisme, il est possible de le traduire en un réseau de simulation en utilisant les blocs de base que nous proposons. Pour chaque transformation proposée, un objet d'émulation a été défini (Figure 6) :

Afin d'assurer la synchronisation entre le modèle d'émulation et de contrôle, la structure d'un élément basique du modèle d'émulation sera donc la suivante (Figure 7) :

1) Un bloc d'entrée,

2) Un premier point de synchronisation, appelé point de pré-synchronisation, en charge du paramétrage de la ressource,

3) Un macro-bloc représentant l'opération réalisée par la ressource, de la même manière que dans un modèle standard,

4) Un second point de synchronisation, appelé point de post-synchronisation, en charge de l'orientation de la pièce vers la prochaine ressource et du déclenchement du travail suivant.

5) Un point de sortie, en charge du routage de l'entité. 


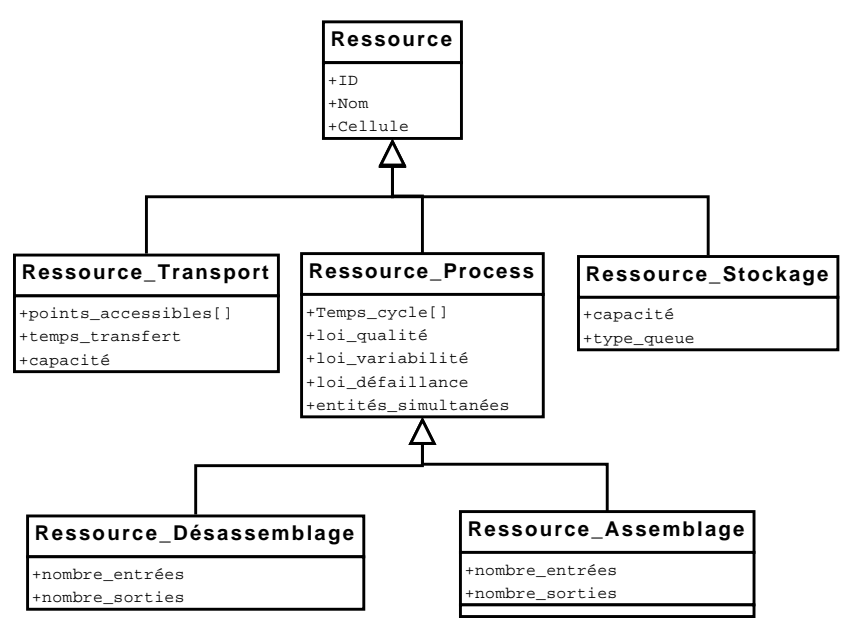

Figure 6. Attributs des objets d'émulation

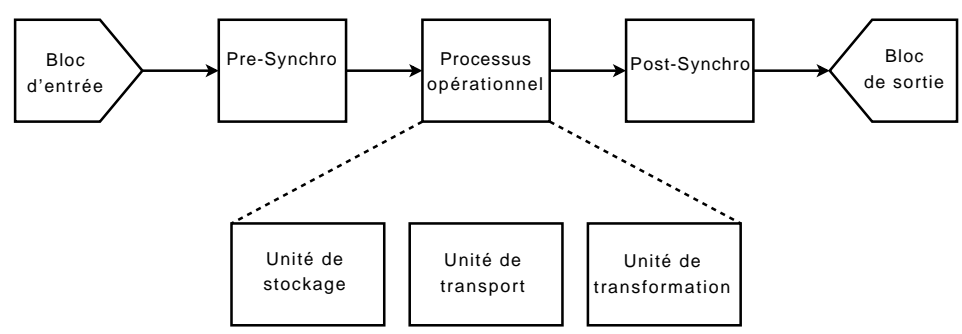

Figure 7. Structure d'un bloc de base du modèle d'émulation

\subsection{Système de contrôle}

Le système de contrôle doit donc réagir à toutes sollicitations du système physique et/ou du modèle d'émulation de celui-ci : il comprendra donc une interface de communication avec ce dernier. Il doit aussi prendre en compte les cadres de décision, contraignant l'autonomie de terrain, issus du progiciel de gestion centralisé : il comprendra donc aussi une interface de communication avec ce dernier.

Pour prendre des décisions, il s'appuiera sur les données issues du système d'information pour générer des scenarii possibles. Il devra les comparer et les analyser afin de choisir une solution à mettre en œuvre : il comprendra donc une base de cas et un système de décision (Figure 8). La base de cas contenant divers scénarii correspondant 

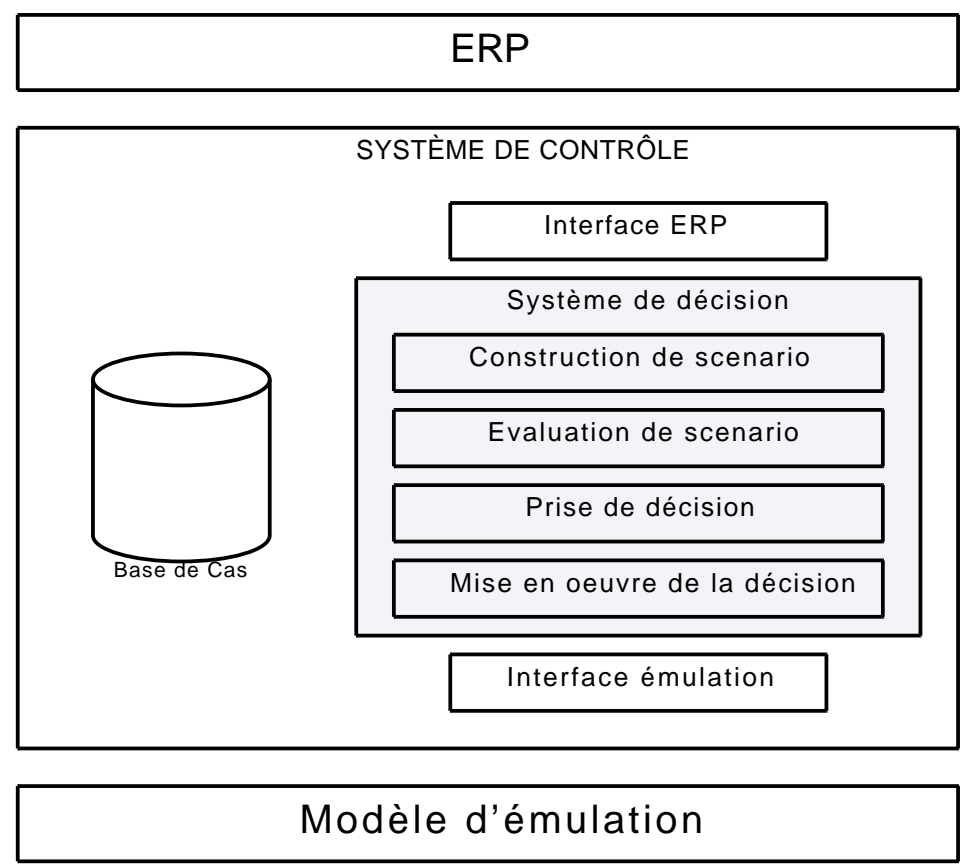

Figure 8. Structure du système de contrôle

à des états du système de production, un état initial typiquement. Notons cependant que pour assurer la généricité de ce système, nous devons pouvoir le remplacer par un autre sans changer l'architecture générale. Pour cela l'interface émulation/contrôle utilise des messages standards contenant les informations suivantes :

- Un identifiant du point de synchronisation (doit permettre d'identifier la ressource, son type et le type de synchronisation : pré ou post, voir Figure 7).

- Un pointeur permettant d'identifier l'entité dans la base de données.

\subsection{Système d'information}

Un système d'information à l'interface entre le modèle d'émulation et le système de contrôle composé d'objets standardisés, assure l'interopérabilité. Il est mis à jour en intégrant les modifications consécutives à une décision prise et en paramétrant le modèle d'émulation en conséquence. Nous distinguons trois types d'événements :

- Les événements qui sont des messages bottom-up en provenance du modèle d'émulation qui déclenchent une mise a jour du système d'information et si nécessaire un processus de prise de décision. Les trames événements sont constituées comme 


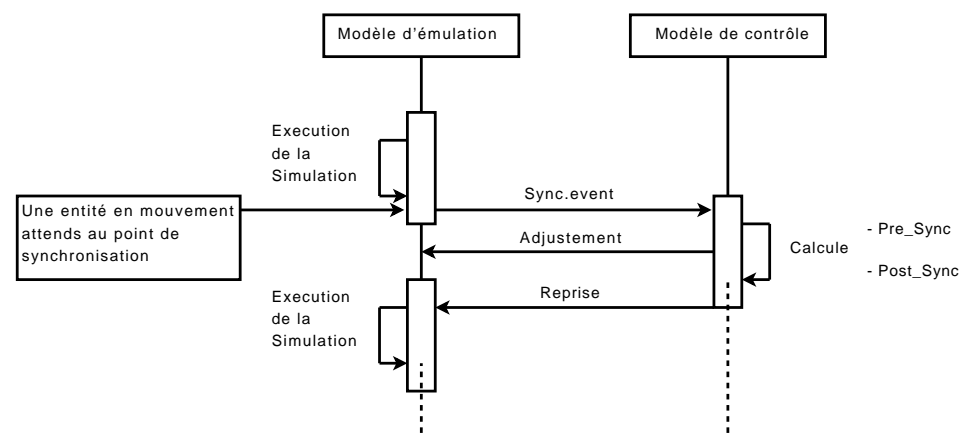

Figure 9. La procedure de synchronisation

suit : horodatage, ressource, ID-produit,

- Les actions, qui sont des messages top-down, sont le résultat d'un processus de prise de décision. Les messages d'action entraînent généralement un paramétrage des blocs ressources du modèle d'émulation, ou le réglage d'un bloc de routage. Une trame d'action contient l'identifiant de la ressource concernée ainsi que la procédure à exécuter et les éventuels paramètres.

- Les ordres de reprise, qui signifient la fin du processus de décision et autorisent la reprise de l'exécution de la simulation.

La procédure de synchronisation (Figure 9) est très simple : lorsqu'une entité atteint un point de synchronisation, un message événement est envoyé au système d'information, portant l'identifiant de l'entité et du point de synchronisation. Le système d'information transmet les changements au système de contrôle qui, si nécessaire, déclenche une procédure de décision. Le modèle d'émulation est mis en pause pendant la durée de cet échange, et l'entité est retenue dans le bloc de synchronisation, jusqu'à réception d'un ordre de reprise.

Le système d'information est donc utilisé comme une interface entre le modèle d'émulation et le système de contrôle. Une approche similaire, basée sur une couche d'échange de données d'après le principe du « tableau noir » est utilisée dans (Monch, 2007). Afin d'assurer la ré-utilisabilité, la généricité et l'interopérabilité, le système d'information (en particulier les données techniques et dynamiques) est construit sur la base des spécifications B2MML et IEC 62264 (Figure 10).

C'est-à-dire :

- Un sous-système de gestion des données techniques.

- Un sous-système de gestion des données dynamiques, contenant un objet pour chaque bloc du modèle d'émulation, et qui a pour rôle de maintenir une image pertinente et cohérente de l'état du système de production. Il est utilisé lors de l'initialisation de la simulation, et également mis à jour tout au long de l'« exécution » afin de 


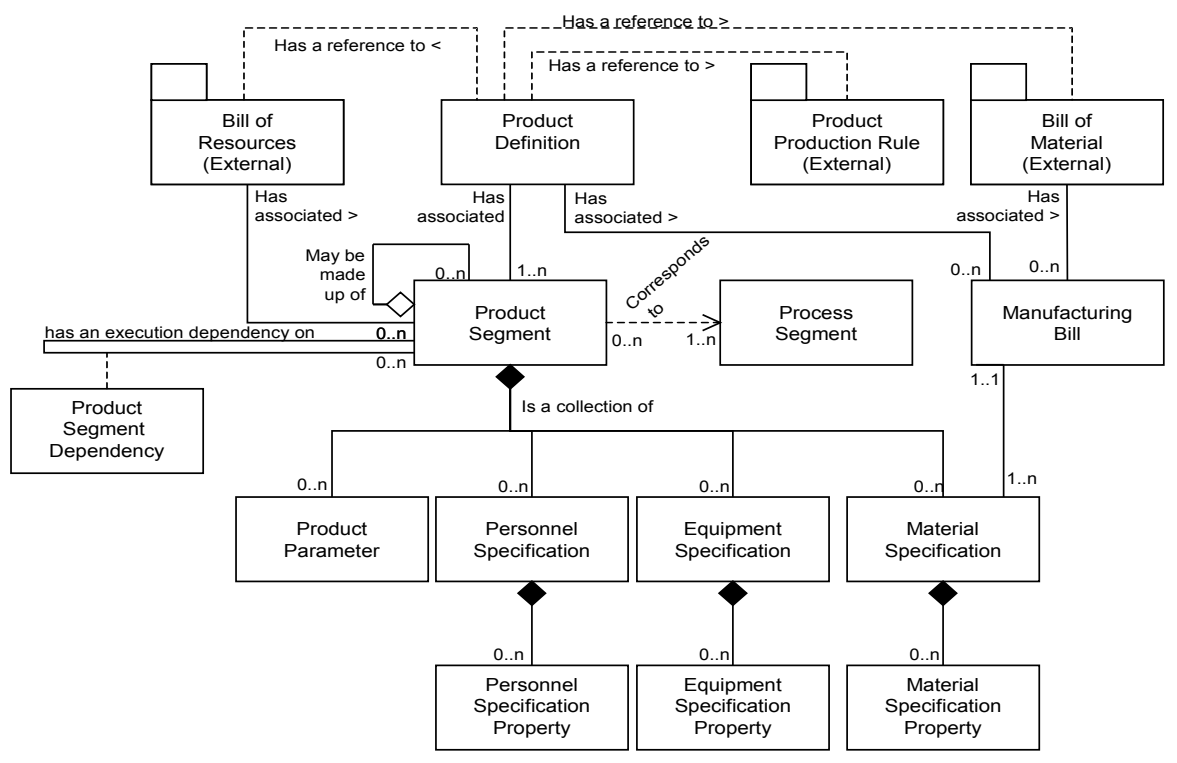

Figure 10. Structure générique du système d'information

suivre l'évolution du système.

- Un sous-système de traçabilité, permettant de mémoriser l'ensemble des événements de production, concernant tant les ressources que les produits, afin d'analyser a posteriori les différentes exécutions.

Plusieurs analyses peuvent être faites à partir des données collectées (des exemples sont présentés dans la section applications). Les blocs d'émulation développés intègrent une fonctionnalité permettant de générer automatiquement le modèle d'information correspondant.

\section{Applications}

Dans ce paragraphe nous présentons les applications particulières que nous avons faites dans les deux entreprises décrites en $§ 2$.

\subsection{Cas du fabricant de meubles en kanban}

Comme nous l'avons présenté dans la deuxième partie, cette entreprise a finalement choisi de piloter ses flux physiques par un système hybride (ERP et kanban). Le système kanban (nommé kanban1 plus loin) est néanmoins particulier car prédéfini à 
l'avance (nombre d'étiquettes et tailles de lots constants; autant d'étiquettes que de postes de charge utiles pour la gamme des pièces à fabriquer). En fait, un système de contrôle calcule et définit le nombre d'étiquettes à placer en début de process de fabrication pour assurer la réalisation du programme hebdomadaire. Une opération terminée sur un poste de charge entraîne le déplacement de l'étiquette sur le poste de charge suivant dans la gamme, donnant ainsi le signal d'autorisation de production pour la suite des opérations. Malgré l'aide apportée par les étiquettes kanban pour le contrôle physique de flux, il en subsiste des difficultés de gestion :

- La gestion des priorités sur les postes de charge n'est pas assurée. En effet, lorsque sur un poste de charge plusieurs étiquettes kanban représentant plusieurs références produits sont présentes, l'opérateur, comme dans tout système kanban classique, ne peut s'appuyer que sur son savoir faire et sur des informations locales pour définir ses priorités.

- L'ordonnancement global n'est donc pas optimal et ne permet pas, par exemple, d'assurer un délai global.(Voir remarque précédente)

- Des pertes de performance en terme de taux d'utilisation des ressources sont constatées, liées à la non-optimalité des décisions localement prises.

- Il n’y a pas de visibilité sur la chaîne logistique interne.

Pour pallier ces faiblesses nous avons proposé une application (nommée kanban2 plus loin) dans laquelle les étiquettes kanban (et non pas les pièces) portent les informations et peuvent donc être à l'initiative de décisions locales. Cette application particulière du concept SCP se concrétise alors par une assistance à la prise de décision dans la gestion des priorités des kanbans (ce sont les « kanbans qui prennent les décisions ») devant les postes de charge. C'est-à-dire que pour initier dans cette entreprise une automatisation des décisions prises « à même le flux physique », nous avons opté pour que ce soit les étiquettes kanbans qui portent les informations nécessaires à la visibilité totale du flux et au choix de priorités entre plusieurs kanbans, à l'endroit considéré. Le système de contrôle implémenté permet donc de reconsidérer l'ordonnancement local en prenant en compte l'impact sur le flux total (jusqu'à l'emballage).

La Figure 11 montre l'architecture spécifique implémentée pour l'évaluation et la démonstration de la mise en œuvre d'un SCP dans cette entreprise. La Figure 12 montre l'adaptation nécessaire du système d'information, en particulier concernant les données dynamiques.

En s'appuyant sur la bibliothèque de cas et la passerelle avec le système de contrôle mise en oeuvre (gestion centralisée de la génération et du placement en début de process de fabrication des étiquettes kanban nécessaires), des situations issues de la réalité ont été reproduites sur le modèle d'émulation. La validation de celui-ci a été menée par analyse de données : sur une période de trois mois de production, nous avons comparé les résultats donnés par le simulateur aux faits réels. Les résultats sur la période couverte par la période de rafraîchissement de l'ordonnancement sont inférieurs à 5 $\%$, en terme de temps d'écoulement, et inférieur à $10 \%$ pour le nombre de palettes 


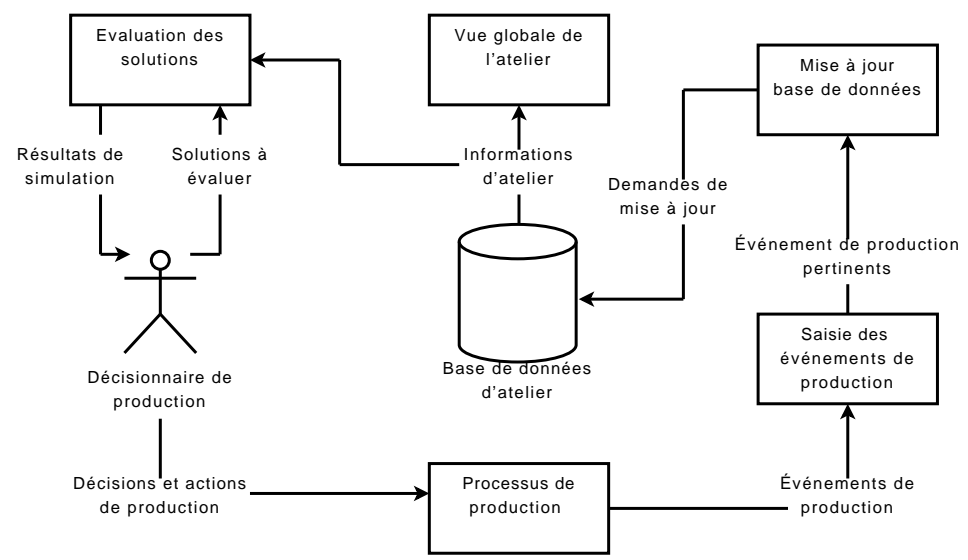

Figure 11. Architecture du système

en-cours. Le modèle d'émulation est donc considéré comme représentatif pour les critères étudiés.

Après validation du modèle d'émulation, trois systèmes de contrôle ont été comparés ( « centralisé », « kanban1 » = kanban qui vient d'être installé dans l'entreprise sans système automatique d'aide à la décision et «kanban 2 » = système proposé de type SCP pour lequel les étiquettes donnent au système de contrôle les informations nécessaires à une aide à la décision). Le système de contrôle a été interfacé avec le modèle d'émulation. Les règles du système kanban2 étant très simples (elles sont strictement régies par procédures), leur intégration s'est faite assez rapidement. L'outil de gestion des étiquettes ajoute en queue de file des étiquettes correspondant à l'ensemble des lots de pièces et ce pour chaque lancement de colis, de sorte que l'ordre FIFO soit respecté sur toutes les machines. La règle de choix d'une tâche pour un centre de charge est également très simple : la première tâche disponible est affectée à la ressource qui vient de se libérer. Une fois la passerelle vers le système de contrôle développée, il nous a été possible de réaliser une étude comparative des deux systèmes de contrôle dans un contexte identique. Les données d'entrée étant issues du système réel, les taux de pannes et de non-qualité sont réalistes.

L'application du concept de SCP (kanban2) à la gestion par kanban semble donc probante au vu de la synthèse des indicateurs de performance représentée dans le tableau 1. Le délai moyen global obtenu avec notre proposition est de $67 \%$ du délai initial avec le système centralisé et de $77 \%$ du délai obtenu avec le kanban classique. Le taux de service client est amélioré de presque 10\%, les ruptures sur la ligne d'emballage (qui rappelons-le est le goulot du système) ont été divisées par 4 et enfin, les stocks d'en-cours (qui rappelons-le sont causes de perte de temps, de désorganisation et de problèmes de qualité) ont été aussi significativement réduits. Ce cas qui constitue un proptotype pour l'entreprise, reste cependant à déployer dans toute celui-ci; en 


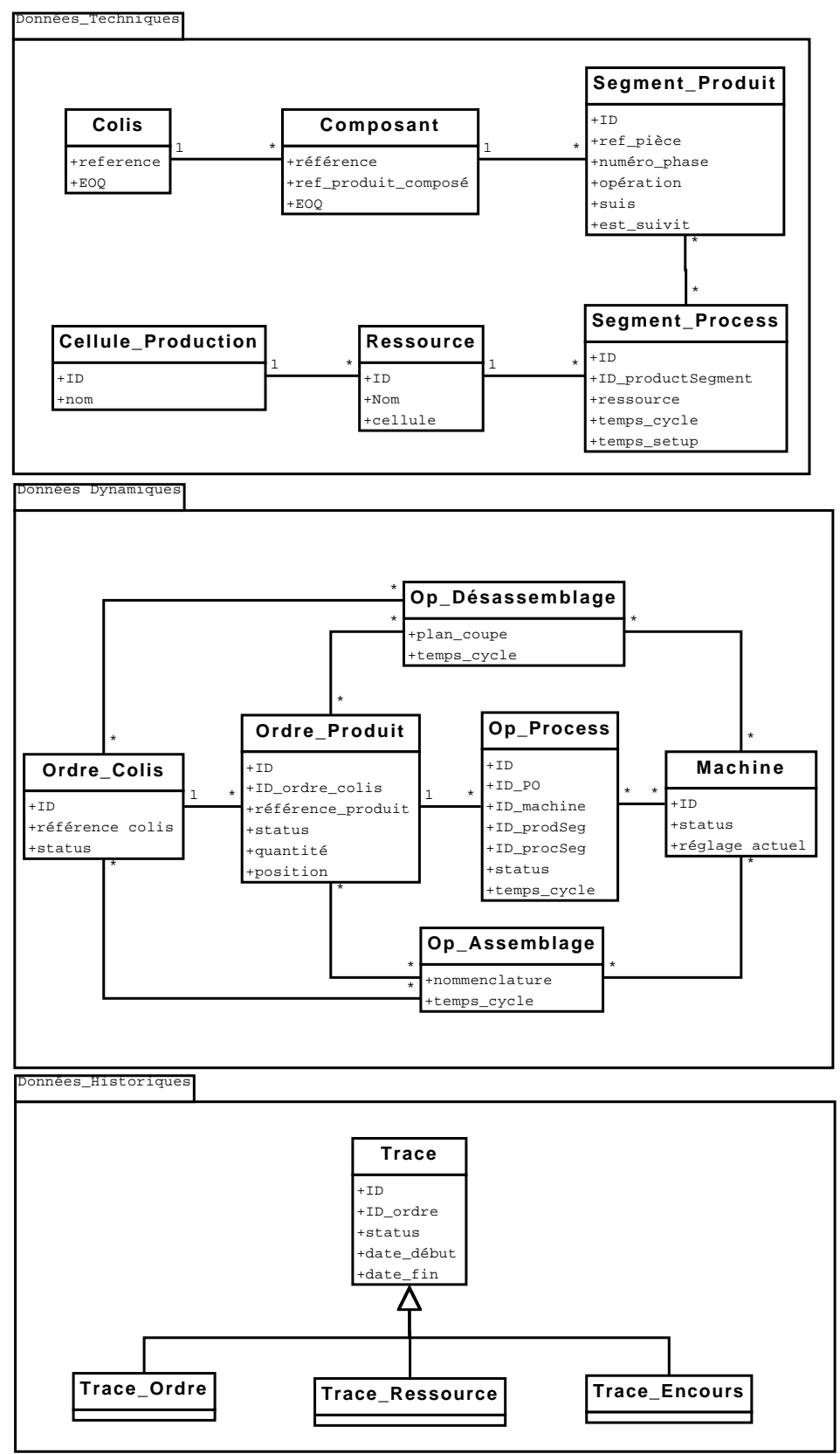

Figure 12. Structure du système d'information 


\begin{tabular}{|l|c|c|c|c|}
\hline Système de contrôle & $\begin{array}{c}\text { Délai } \\
\text { moyen }\end{array}$ & $\begin{array}{c}\text { Taux de } \\
\text { service }(\%)\end{array}$ & $\begin{array}{c}\text { Retard } \\
\text { maximal }\end{array}$ & $\begin{array}{c}\text { Rupture } \\
\text { emballage (h) }\end{array}$ \\
\hline \hline Centralisé & 40 & 72 & 10 & 4 \\
\hline Kanban 1 & 35 & 78 & 7 & 3 \\
\hline Kanban 2 & 27 & 81 & 6 & 1 \\
\hline
\end{tabular}

\begin{tabular}{|l|c|c|c|}
\hline Système de contrôle & $\begin{array}{c}\text { Stock moyen } \\
\text { WIP1 (palettes) }\end{array}$ & $\begin{array}{c}\text { Stock moyen } \\
\text { WIP2 (palettes) }\end{array}$ & $\begin{array}{c}\text { Stock moyen } \\
\text { WIP3 (palettes) }\end{array}$ \\
\hline \hline Centralisé & 130 & 169 & 403 \\
\hline Kanban 1 & 115 & 160 & 350 \\
\hline Kanban 2 & 120 & 147 & 371 \\
\hline
\end{tabular}

Tableau 1. Synthèse des résultats de l'étude

particulier dans des processus où des décisions de priorités entre différentes lignes de produits (donc entre différentes boucles kanban) sont, par exemple, à prendre.

\subsection{Cas du fabricant de climatiseurs en DFT}

Dans cette entreprise un pilote a été choisi pour implémenter l'approche SCP. Il s'agit d'une ligne d'alimentation de 4 postes de travail alimentant deux lignes d'assemblage dans deux usines différentes (Figure 13). Issu de l'ERP, le Programme Directeur de Production (PDP) donne le travail hebdomadaire de la ligne d'assemblage du site A. Ce PDP est le niveau le plus bas du système de gestion centralisé (ERP). Des Ordres de Fabrication (OF) sont aussi envoyés par anticipation à la ligne d'alimentation afin d'assurer la disponibilité des composants. La gestion distribuée de cette ligne d'alimentation est nécessitée par la volonté de réduire les stocks aux points de synchronisation (IPK). Lors de la phase d'évaluation de pertinence du SCP sur ce pilote, il a été nécessaire d'émuler diverses hypothèses de positionnement des points de transmission d'informations des produits vers le système de contrôle, car ceux-ci avaient un impact sur les indicateurs de performance (délais, niveaux de stock d'en-cours, etc.), ce que nous montrons par la suite en terme d'application.

La Figure 14 montre l'architecture installée qui utilise la technologie Internet pour communiquer entre les usines et les postes de travail et la Figure 15 décrit la structure du système d'information adaptée à ce cas d'étude. Celles-ci sont aussi déduites de la modélisation générique proposée dans le paragraphe 4.2.

Les différentes hypothèses $(\mathrm{H} 1=$ le lecteur est installé en tête de ligne d'assemblage, $\mathrm{H} 2$ = lecteur installé au poste $2, \mathrm{H} 3=$ lecteur installé au poste $3, \mathrm{H} 4=$ lecteur installé au point d'IPK.) de position d'implantation des lecteurs RFID ont été testées pour mesurer l'impact de cette position sur différentes indicateurs tels les stocks en 


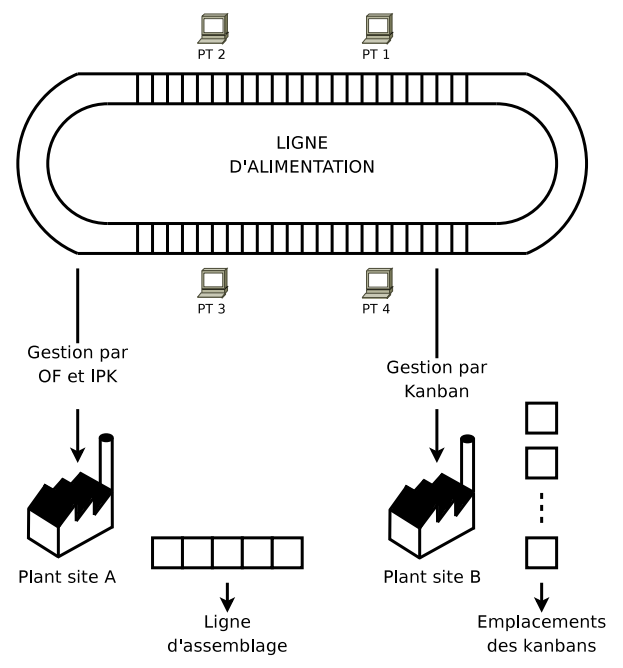

Figure 13. Vue du pilote

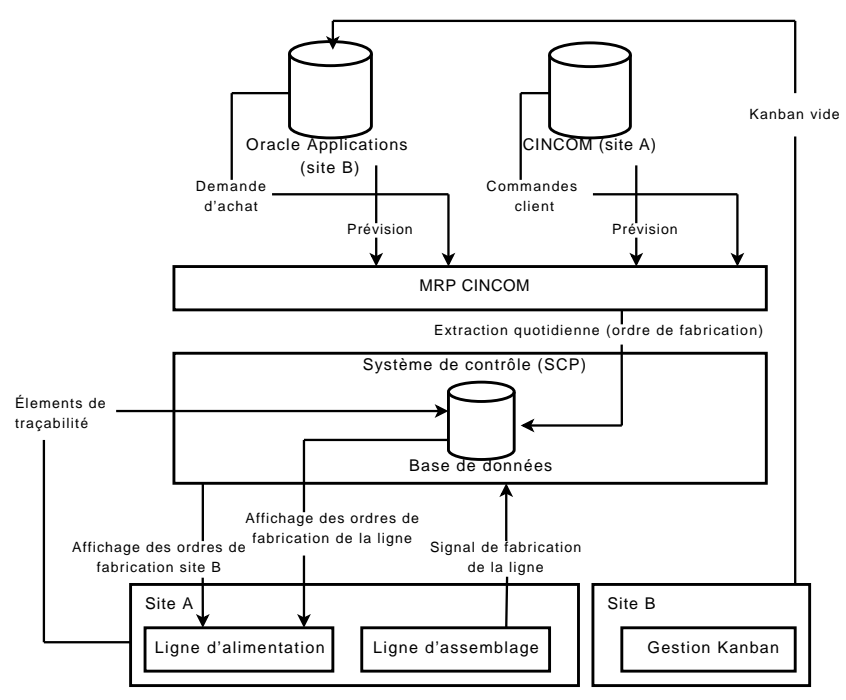

Figure 14. Architecture générale du système pour les lignes d'assemblage en DFT

IPK, les délais, etc. La Figure 16 montre les deux hypothèses extrêmes $(\mathrm{H} 1=$ lecteur installé à la tête de ligne, H4 = lecteur installé au point d'IPK). 


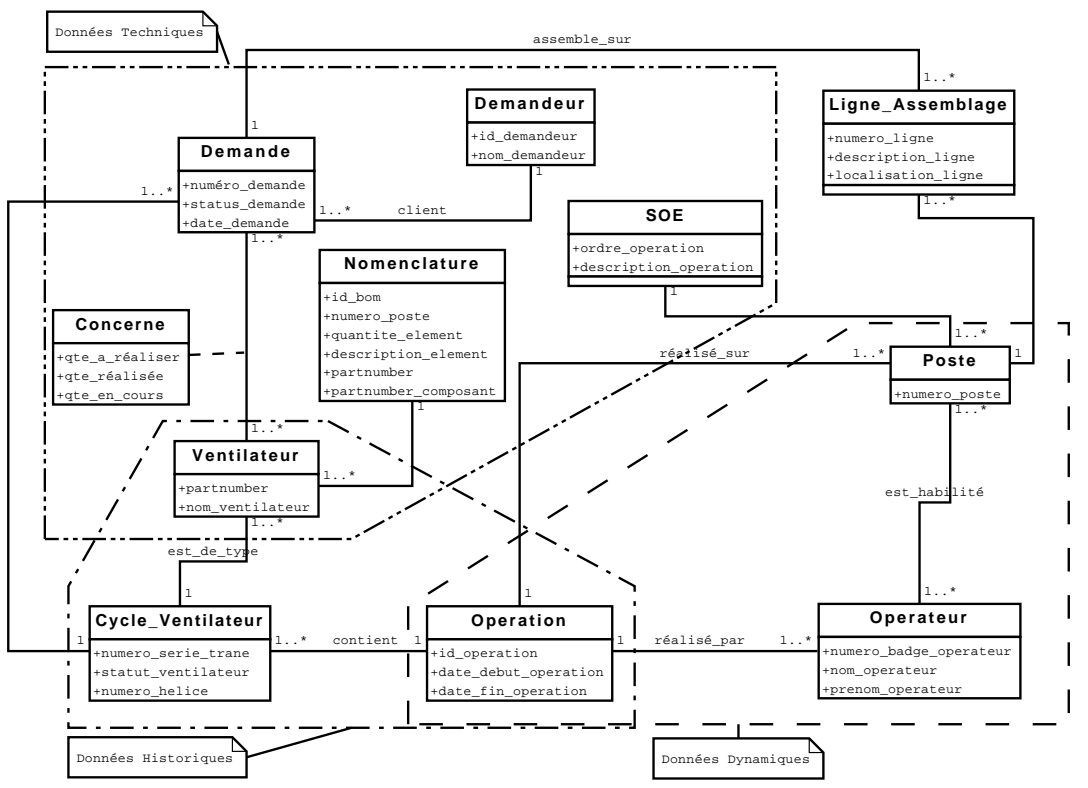

Figure 15. Structure du système d'information

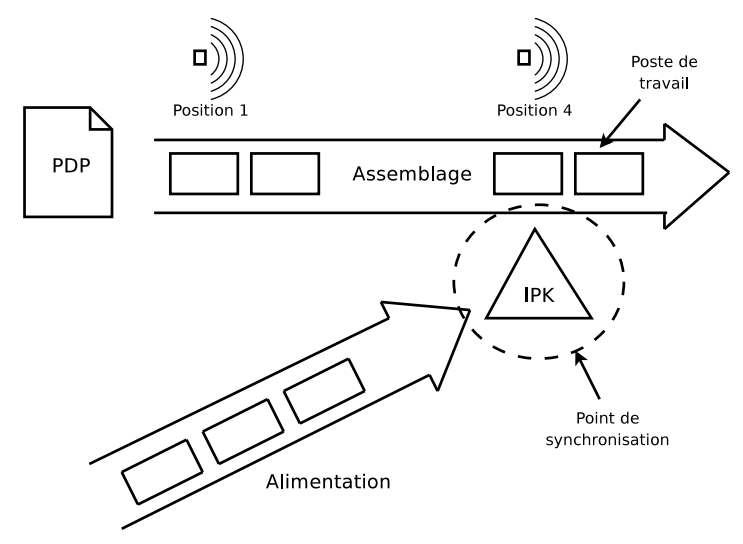

Figure 16. Les scénarii de positionnement des lecteurs

Les Figures 17 a et $\mathrm{b}$ montrent respectivement les résultats sur l'indicateur délai en minutes par composant sur la ligne d'alimentation et sur le produit fini ('indicateur stock à aussi été testé). Les analyses sont à observer uniquement dans la zone A pour laquelle le système a atteint son rythme nominal. Aucun écart n'est constaté 
sur la ligne d'assemblage qui dans les deux cas a réalisé son PDP. Par contre, sur la ligne d'alimentation l'hypothèse $\mathrm{H} 2$ permet de minimiser les délais d'obtention et les niveaux de stock des IPK (ligne pointillé Figure 17b).
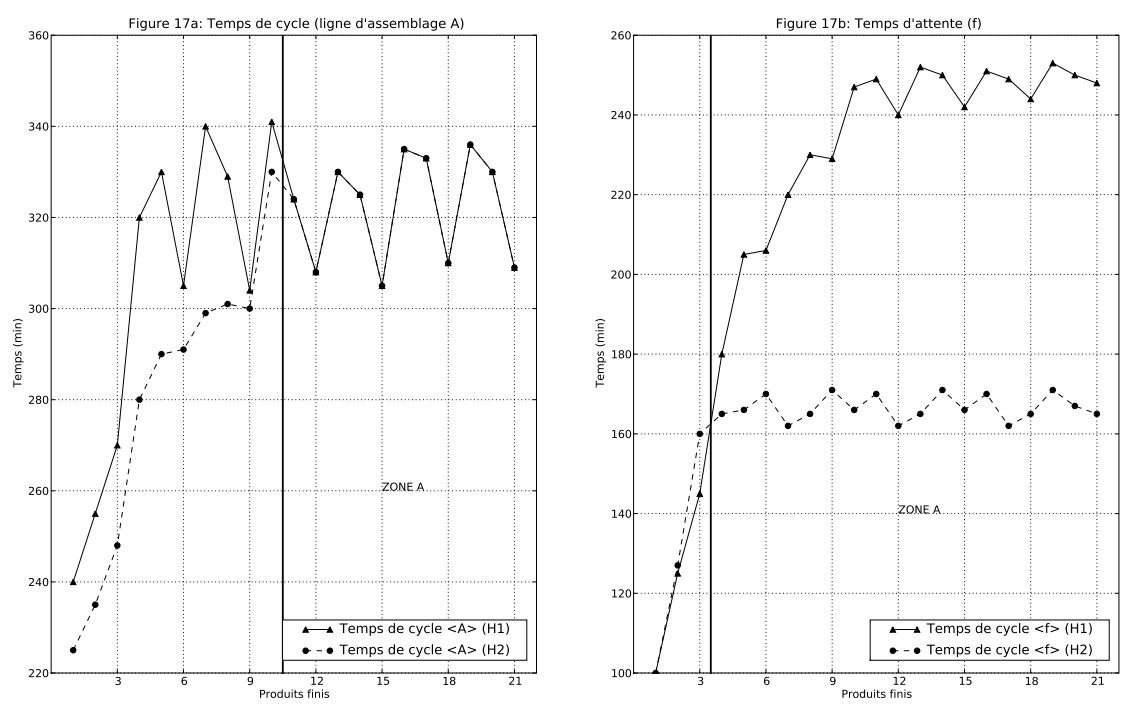

Figure 17. Résultats d'analyse

Ce pilote permet de montrer que l'information portée par le produit, dans ce contexte de JiT en DFT et par l'information anticipée « du juste nécessaire» qu'il donne aux opérateurs, offre la possibilité d'ajuster en dynamique les ordres de fabrication des composants sur la ligne d'alimentation et ainsi conduit à la minimisation des stocks IPK de synchronisation. En effet, dans l'hypothèse 1 où le lecteur est en tête de ligne d'assemblage (position 1), le système est dans une configuration équivalente au système centralisé sans lecteur, mais pour lequel l'information serait donnée par des OF issus de l'ERP. Dans ce cas donc le SCP n'apporterait évidemment rien. A l'opposé, si le lecteur est au point d'IPK (position 2), il faudrait être extrêmement réactif, le taux de service serait probablement dégradé. Les scénarii d'émulation ont permis donc de trouver le bon emplacement qui maximise les indicateurs de performance tout en minimisant le nombre de lecteurs.

Ceci étant fait, il est désormais possible de faire prendre les décisions au système de contrôle intégré à la base de données (fonction principale du SCP). En effet, cette dernière centralise les informations issues du site B par le flux de retour des kanbans vides qui transitent par la base Oracle, d'une part, alors que les informations terrain issues du site A, y reviennent directement via les lecteurs RFID, d'autre part. Cepen- 
dant, à l'heure actuelle, ce système est perfectible car seules les règles utilisées par les opérateurs ont été programmées : aucune optimisation n'a été réalisée.

\section{Perspectives}

Dans ce contexte de décisions locales autonomes et de très court terme, nous avons comme perspectives de proposer une approche d'optimisation qui s'intègre dans ce cadre d'une démarche de juste à temps et d'un SCP. Il s'agit de prendre en compte en ligne des informations portées par le produit ou par une entité du système physique (RFID sur une pièce ou sur un Kanban) et de les considérer dans l'optimisation des problèmes de décision. Ainsi pour chacun des cas industriels posés, nous pouvons d'ores et déjà décrire les problèmes de décision correspondant et rapporter des travaux proches de la littérature.

Concernant le fabricant de climatiseurs, une première problématique concerne la synchronisation de la ligne principale en prenant en compte plusieurs lignes d'approvisionnement. Ce problème relève de l'équilibrage de lignes d'assemblage (ALB) avec la particularité de considérer des lignes d'alimentation. Pour ce type de problèmes, plusieurs revues de la littérature ont été suggérées, nous citons (Buxey et al., 1973), (Ghosh et al., 1989) et (Scholl et al., 2006). Plus récemment, une classification des problèmes de type ALB est proposée dans (Boysen et al., 2006). Cette étude révèle le gap existant entre les problèmes considérés dans le monde académique et ceux qui se posent dans la réalité industrielle. En effet, de nombreux travaux de la littérature s'intéressent à des problèmes théoriques de type SALBP avec des hypothèses très restrictives considérant, par exemple, que les opérations ont un seul mode d'exécution. En pratique, c'est rarement le cas car il existe souvent plusieurs alternatives et notamment, en phase de conception, si on envisage plusieurs types d'équipements possibles. Chaque type d'équipement engendre un coût différent qui doit être pris en compte lors de la prise de décision (Belmokhtar et al., 2006).

L'étude bibliographique menée jusqu'à ce jour nous a permis de recenser les travaux de (Tempelmeier, 2003) qui considèrent que l'équilibrage d'une ligne principale intègre celui de lignes d'approvisionnement. (Lapierre et al., 2004) considèrent également des lignes d'alimentation et suggèrent d'équilibrer la ligne principale dans un premier temps puis sur la base du temps de cycle principal obtenu, ils réalisent l'équilibrage des lignes d'alimentation en supposant que les opérateurs peuvent intervenir sur le point de croisement de la ligne principale et des lignes d'alimentations (même contexte que pour la DFT).

Concernant le fabricant de meubles, les problèmes posés sont liés à la détermination d'un ordre de passage des produits devant des machines (stations) dans un contexte de juste à temps, l'ensemble des travaux de la littérature suggèrent des approches de simulation en appliquant de simples règles de priorité. En particulier, (Hum et al., 1997) évaluent plusieurs règles basées sur le nombre de kanbans pour définir l'ordre des produits en attente devant les stations. (Vinod et al., 2007) suggèrent de 
nouvelles règles de priorité basées sur les temps de préparations pour un problème de job shop avec temps de préparation dépendant de la séquence. Les auteurs mènent une étude expérimentale sous des conditions liées à la charge de l'atelier, ratios des temps de préparations et aux dates échues.

Dans les travaux cités, il s'agit de résoudre des problèmes hors-ligne c'est-à-dire sans la prise en compte d'événements liés au fonctionnement du système en temps réel.(Artigues et al., 2001) suggèrent une approche temps réel pour les problèmes d'ordonnancement d'ateliers travaillant à la commande. Une des pistes que nous pensons prometteuse est d'intégrer les informations portées par le produit dans une telle approche pour déterminer l'ordre de passage des produits dans un atelier, d'une part, et de synchroniser des lignes d'assemblage en présence de lignes d'approvisionnement, d'autre part.

\section{Conclusion}

Dans cet article, nous avons pu montrer en quoi le concept de Système Contrôlé par le Produit (SCP) pouvait être pertinent pour l'automatisation de ou pour l'assistance à la décision locale (à même le flux de produits) dans un contexte de Juste à Temps (JiT), d'une part, mais aussi pour la conception et l'évaluation de tels systèmes, d'autre part. Ces derniers étant par définition des systèmes hybrides, à savoir, des systèmes dans lesquels des décisions indépendantes sont prises sur le terrain à l'intérieur d'un cadre d'autonomie autorisé par le système centralisé.

Nous avons aussi proposé une architecture permettant la mise en oeuvre de ce concept SCP dans un tel contexte de JiT, cette architecture comprenant un système de contrôle, un émulateur, une base de données et des interfaces adaptées.

Dans un autre article (Pannequin et al., 2009) nous avons proposé une méthodologie de déploiement de ce type d'architecture dans divers contextes industriels.

En termes de perspectives, nous avons entamé une réflexion sur l'identification des problèmes de décision à intégrer et sur la manière de faire évoluer les approches de la littérature afin de prendre en compte des informations portées par le produit. Pour les deux cas pratiques étudiés dans cet article, il s'agit de définir l'ordre de passage des produits sur les stations (cas du fabricant de meubles) et d'équilibrer une ligne d'assemblage en considérant des lignes d'approvisionnement (cas du fabricant de climatiseurs).

\section{Bibliographie}

Artigues C., Roubellat F., Ordonnancement d'ateliers en temps réel : Ordonnancement de la production, Hermes Science Publications, Paris, 2001.

Babiceanu R., Chen F., « Development and Applications of Holonic Manufacturing Systems : A Survey », Journal of Intelligent Manufacturing, vol. 17, n 1, p. 111-131, February, 2006. 
Belmokhtar S., Dolgui A., Guschinsky N., Levin G., « Integer programming models for logical layout design of modular machining lines », Computers \& industrial engineering, vol. 51, p. 502-518, 2006.

Bongaerts L., Monostori L., Mcfarlane D., Kadar B., « Hierarchy in distributed shop floor control », Computers in Industry, vol. 43, n² 2, p. 123-137, October, 2000.

Boysen N., Fliedner M., Scholl A., « A classification of assembly line balancing problems », European Journal of Operational Research, vol. 183, p. 674-693, 2006.

Brennan R. W., « Performance comparison and analysis of reactive and planning-based control architectures for manufacturing », Robotics and Computer-Integrated Manufacturing, vol. 16, n²-3, p. 191-200, April, 2000.

Burbidge J., Falster P., Riis J., Svendsen O., « Integration in Manufacturing », Computers in industry, vol. 9, $\mathrm{n}^{\circ}$ 4, p. 297-305, 1987.

Buxey G., Slack N. D., Wild N., « Production flow line system design-a review », AIIE Transactions, vol. 5, p. 37-48, 1973.

Cavalieri S., Macchi M., P V., « Benchmarking the performance of manufacturing control system : design principles for a web based simulated testbed », Journal of Intelligent Manufacturing, vol. 14, $\mathrm{n}^{\circ}$ 1, p. 43-58, 2003.

Costanza J., Just-In-Time manufacturing, 3rd edn, excellence John Costanza Institute of Technology Inc, 1996.

El Haouzi H., Thomas A., « A Methodological Approach to Build Simulation Models of Manufacturing Systems with Distributed Control », Interna-tional Conference on Industrial Engineering and Systems Management, Marrakech, Morocco., 2005.

Ghosh S., Gagnon R. J., « A comprehensive literature review and analysis of the design, balancing and scheduling of assembly systems », International Journal of Production Research, vol. 4, p. 637-670, 1989.

Gouyon D., Contrôle par le produit des systèmes d'exécution de la production : apport des techniques de synthèse, PhD thesis, Université Heri Poincaré, Nancy I, December, 2004.

Hum S. H., Lee C. H., « JIT Scheduling Rules : A Simulation Evaluation », International Journal of Management Science, vol. 26, n 3, p. 381-395, 1997.

Koestler A., The Ghost in the Machine, Hutchinson \& Co Ltd., London, UK, March, 1976.

Lapierre S. D., Ruiz A. B., « Balancing assembly lines : An industrial case study », Journal of the Operational Research Society, vol. 55, p. 589-597, 2004.

Le Moigne J. L., La théorie du système général. Théorie de la modélisation, Presse Universitaires de France, 1977. réédition de 1994. ISBN 2130384838.

Monch L., « Simulation-based benchmarking of production control schemes for complex manufacturing systems », Control Engineering Practice, vol. 15, n 11, p. 1381-1393, 2007.

Morel G., Panetto H., Zaremba M., Mayer F., « Manufacturing Enterprise Control and Management System Engineering : paradigms and open issues », Annual Reviews in Control, vol. 27, $\mathrm{n}^{\circ}$ 2, p. 199-209, 2003.

Morel G., Valckenaers P., Faure J.-M., Pereira C. E., Dietrich C., « Manufacturing plan control challenges and isssues », Control Engineering Pratice, vol. 15, n 11, p. 1321-1331, 2007.

Pannequin R., Morel G., Thomas A., « The performance of product-driven manufacturing control : An emulation-based benchmarking study », Computer in Industry, 2009. 
Pannequin R., Thomas A., « Centralized versus distributed decision, an industrial case », 11th IFAC Symposium on Information and Control Problem in Manufacturing INCOM'2004, 2004.

Scholl A., Becker C., « State-of-the-art exact and heuristic solution procedure for simple assembly line balancing », European Journal of Operational Research, vol. 168, $n^{\circ}$ 3, p. 666-693, 2006.

Scholz-Reiter B., Gorldt C., Hinrichs U., « Simulation of a RFID-based KANBAN System for the Production Industries », PPS MANAGEMENT, vol. 13(2), p. 16-19, 2008.

Tempelmeier H., « Pratical considerations in the optimization of flow production systems », International Journal of Production Research, vol. 41, p. 149-170, 2003.

Valckenaers P., Van Brussel H., Bongaerts L., Wyns J., « Holonic manufacturing systems », Integr. Comput.-Aided Eng., vol. 4, n 3, p. 191-201, 1997.

Vinod V., Sridharan R., " Scheduling a dynamic job shop production system with sequencedependent setups : An experimental study », Robotics and Computer-Integrated Manufacturing, 2007. 